\title{
Non-contiguous finished genome sequence and description of Alistipes timonensis sp. nov.
}

\author{
Jean-Christophe Lagier', Fabrice Armougom ${ }^{1}$, Ajay Kumar Mishra', Thi-Tien Nguyen ${ }^{1}$, \\ Didier Raoult ${ }^{1}$ and Pierre-Edouard Fournier ${ }^{1 *}$ \\ ${ }^{1}$ Unité de Recherche sur les Maladies Infectieuses et Tropicales Emergentes, UMR, \\ Aix-Marseille Université, Marseille, France \\ *Corresponding author: Pierre-Edouard Fournier (pierre-edouard.fournier@univmed.fr)
}

Keywords: Alistipes timonensis, genome

Alistipes timonensis strain $\mathrm{JC} 136^{\mathrm{T}} \mathrm{sp}$. nov. is the type strain of $A$. timonensis sp. nov., a new species within the genus Alistipes. This strain, whose genome is described here, was isolated from the fecal flora of a healthy patient. A. timonensis is an obligate anaerobic rod. Here we describe the features of this organism, together with the complete genome sequence and annotation. The 3,497,779 bp long genome (one chromosome but no plasmid) contains 2,742 protein-coding and 50 RNA genes, including three rRNA genes.

\section{Introduction}

Alistipes timonensis strain JC136 ${ }^{\mathrm{T}}$ (= CSUR P148 = DSM 25383) is the type strain of $A$. timonensis sp. nov. This bacterium is a Gram-negative, anaerobic, indole-positive bacillus and was isolated from the stool of a healthy Senegalese patient as part of a "culturomics" study aiming at cultivating individually all species within human feces.

With more than 3,000 genome sequences available, bacterial genomics has revolutionized several aspects of microbiology. To date, taxonomy has remained unaffected by this progress, despite the debate around the definition of bacterial species. Despite its elevated cost, poor reproducibility and inter-laboratory comparability, DNA-DNA hybridization remains the "gold standard" criterion [1]. Even the application of internationally validated cutoff values in $16 \mathrm{~S}$ rRNA sequence similarity that enabled the taxonomic classification or reclassification of hundreds of taxa, is debated [2]. High throughput genome sequencing and mass spectrometric analyses of bacteria provide access to a wealth of genetic and proteomic information [3]. We propose to use a polyphasic approach [4] to describe new bacterial taxa that includes their genome sequence, MALDI-TOF spectrum and main phenotypic characteristics (habitat, Gram-stain reaction, culture and metabolic characteristics, and when applicable, pathogenicity).
Here we present a summary classification and a set of features for $A$. timonensis sp. nov. strain JC136 ${ }^{\mathrm{T}}$ together with the description of the complete genomic sequencing and annotation. These characteristics support the circumscription of the species A. timonensis.

The genus Alistipes (Rautio et al. 2003) was created in 2003 [5]. To date, this genus, composed of bile-resistant, strictly anaerobic and Gramnegative bacilli, contains five species including $A$. finegoldii (Rautio et al. 2003) [5], A.indistinctus (Nagai et al. 2010) [6], A. onderdonkii (Song et al. 2006) [7], A. putredinis (Weinberg et al. 1937) Rautio et al. 2003 [5], and A. shahii (Song et al. 2006) [7]. Pigment production, initially considered as characteristic of Alistipes species, was recently demonstrated to be inconstant [8]. Members of the genus Alistipes are members of the normal human intestinal microbiota, but have also been reported in urine and the mouth [7], and have occasionally been isolated from abdominal, appendiceal and rectal abscesses, blood cultures from colon cancer patients [9], and feces from children with irritable bowel syndrome [10]. A. putredinis was also demonstrated to be associated to cruciferous vegetable intake [11]. In addition, $A$. finegoldii has been suspected to play the role of growth promoter in chickens [12]. 


\section{Classification and features}

A stool sample was collected from a healthy 16year-old male Senegalese volunteer patient living in Dielmo (a rural village in the Guinean-Sudanian zone in Senegal), who was included in a research protocol. The patient gave an informed and signed consent, and the agreement of the National Ethics Committee of Senegal and the local ethics committee of the IFR48 (Marseille, France) were obtained under agreement 09-022. The fecal specimen was preserved at $-80^{\circ} \mathrm{C}$ after collection and sent to Marseille. Strain JC136 (Table 1) was isolated in June 2011 by anaerobic cultivation on $5 \%$ sheep blood-enriched Columbia agar (BioMerieux, Marcy l'Etoile, France). This strain exhibited $96.98 \%$ and 98.13\% nucleotide sequence similarities with $A$. shahii (Song et al. 2006) and A. senegalensis (Mishra et al. 2012), respectively, the phylogenetically closest validated Alistipes species (Figure 1) [7]. This value was lower than the $98.7 \% 16 \mathrm{~S}$ rRNA gene sequence threshold recommended by Stackebrandt and Ebers to delineate a new species without carrying out DNA-DNA hybridization [2]. It should be noted that both $A$. senegalensis strain JC50T and strain JC136 were cultivated from the same individual.

Table 1. Classification and general features of Alistipes timonensis strain JC136 ${ }^{\top}$

\begin{tabular}{|c|c|c|c|}
\hline MIGS ID & Property & Term & Evidence code $^{\mathrm{a}}$ \\
\hline & \multirow{8}{*}{ Current classification } & Domain Bacteria & TAS [13] \\
\hline & & Phylum Bacteroidetes & TAS $[14,15]$ \\
\hline & & Class Bacteroidia & TAS $[14,16]$ \\
\hline & & Order Bacteroidales & TAS $[14,17]$ \\
\hline & & Family Rikenellaceae & TAS $[14,18]$ \\
\hline & & Genus Alistipes & TAS $[5,19]$ \\
\hline & & Species Alistipes timonensis & IDA \\
\hline & & Type strain JC136 ${ }^{\top}$ & IDA \\
\hline & Gram stain & Negative & IDA \\
\hline & Cell shape & Bacilli & IDA \\
\hline & Motility & Nonmotile & IDA \\
\hline & Sporulation & Nonsporulating & IDA \\
\hline & Temperature range & Mesophile & IDA \\
\hline & Optimum temperature & $37^{\circ} \mathrm{C}$ & IDA \\
\hline MIGS-6.3 & Salinity & Growth in $\mathrm{BHI}$ medium $+1 \% \mathrm{NaCl}$ & IDA \\
\hline \multirow[t]{3}{*}{ MIGS-22 } & Oxygen requirement & Anaerobic & IDA \\
\hline & Carbon source & Unknown & NAS \\
\hline & Energy source & Unknown & NAS \\
\hline MIGS-6 & Habitat & Human gut & IDA \\
\hline \multirow[t]{3}{*}{ MIGS-15 } & Biotic relationship & Free living & IDA \\
\hline & Pathogenicity & Unknown & \\
\hline & Biosafety level & 2 & \\
\hline MIGS-14 & Isolation & Human feces & NAS \\
\hline MIGS-4 & Geographic location & Senegal & IDA \\
\hline MIGS-5 & Sample collection time & September 2010 & IDA \\
\hline MIGS-4.1 & Latitude & 13.7167 & IDA \\
\hline MIGS-4.1 & Longitude & 16.4167 & IDA \\
\hline MIGS-4.3 & Depth & Surface & IDA \\
\hline MIGS-4.4 & Altitude & $51 \mathrm{~m}$ above sea level & IDA \\
\hline
\end{tabular}

Evidence codes - IDA: Inferred from Direct Assay; TAS: Traceable Author Statement (i.e., a direct report exists in the literature); NAS: Non-traceable Author Statement (i.e., not directly observed for the living, isolated sample, but based on a generally accepted property for the species, or anecdotal evidence). These evidence codes are from the Gene Ontology project [20]. If the evidence is IDA, then the property was directly observed for a live isolate by one of the authors or an expert mentioned in the acknowledgements. 


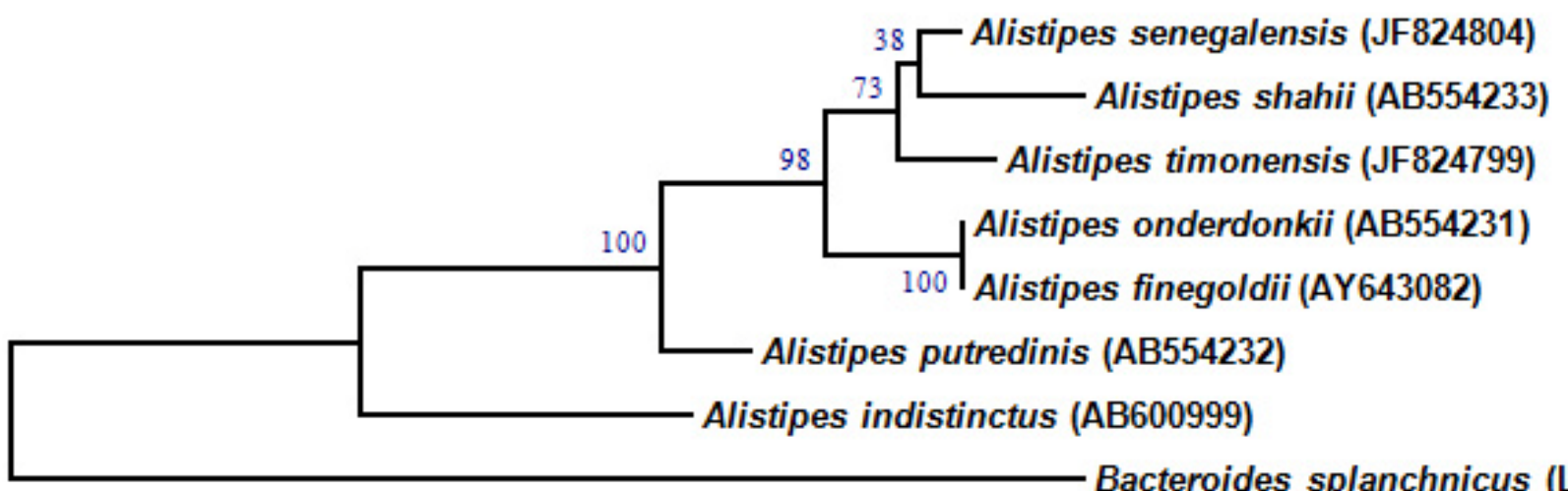

Bacteroides splanchnicus (L16496)

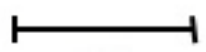

Figure 1. Phylogenetic tree highlighting the position of Alistipes timonensis strain $\mathrm{JC} 136^{\top}$ relative to other type strains within the Alistipes genus. GenBank accession numbers are indicated in parentheses. Sequences were aligned using CLUSTALW, and phylogenetic inferences obtained using the maximum-likelihood method within the MEGA software. Numbers at the nodes are percentages of bootstrap values obtained by repeating the analysis 500 times to generate a majority consensus tree. Porphyromonas asaccharolytica was used as an outgroup. The scale bar represents a $2 \%$ nucleotide sequence divergence.

Different growth temperatures $\left(25,30,37,45^{\circ} \mathrm{C}\right)$ were tested; no growth occurred at $25^{\circ} \mathrm{C}$ and $45^{\circ} \mathrm{C}$, growth occurred at $30^{\circ} \mathrm{C}$, and optimal growth was observed at $37^{\circ} \mathrm{C}$. Colonies were $0.2 \mathrm{~mm}$ to 0.3 $\mathrm{mm}$ in diameter on blood-enriched Columbia agar and Brain Heart Infusion (BHI) agar. Growth of the strain was tested under anaerobic and microaerophilic conditions using GENbag anaer and GENbag microaer systems, respectively
(BioMerieux), and in the presence of air, with or without of $5 \% \mathrm{CO}_{2}$, and in aerobic conditions. Optimal growth was achieved anaerobically. No growth was observed in aerobic, microaerophilic and $5 \% \mathrm{CO}_{2}$ atmospheres. Gram staining showed Gram negative rods (Figure 2). A motility test was negative. Cells grown on agar have a mean diameter of $0.62 \mu \mathrm{m}$ (Figure 3 ) and produce brown pigment.

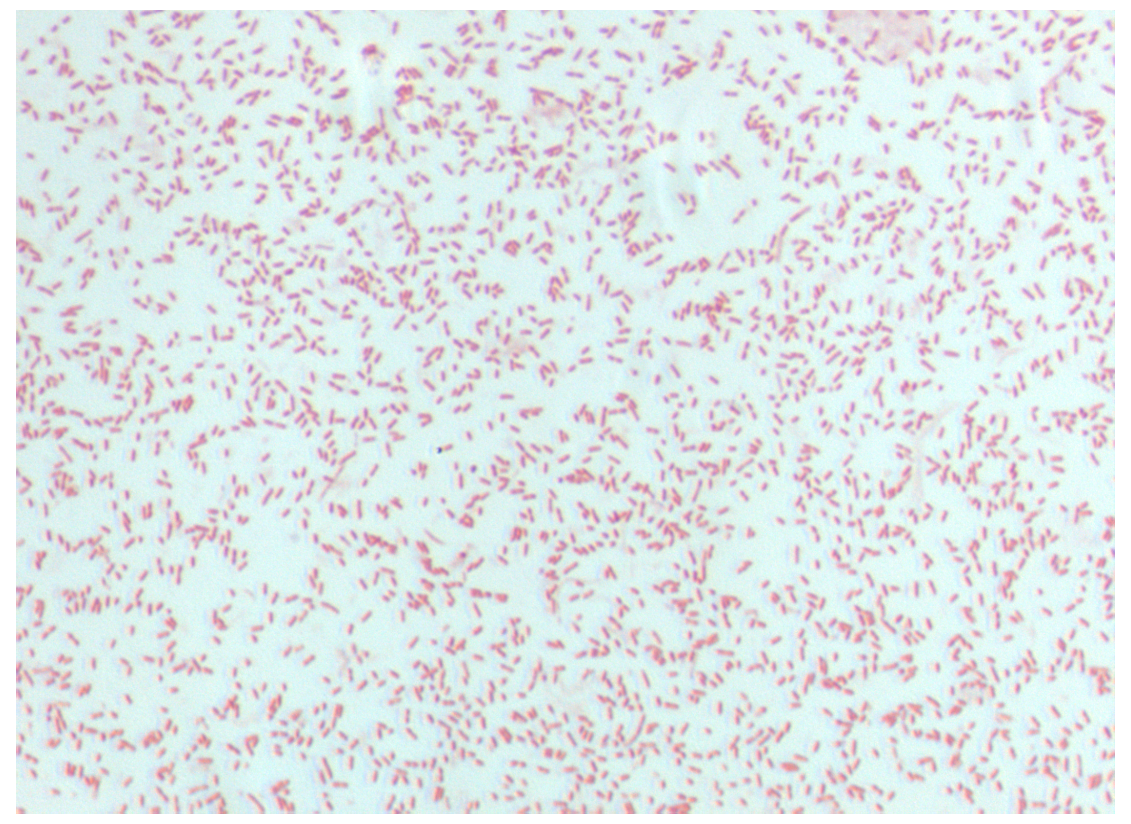

Figure 2. Gram staining of $A$. timonensis strain $\mathrm{JC} 136^{\top}$ 


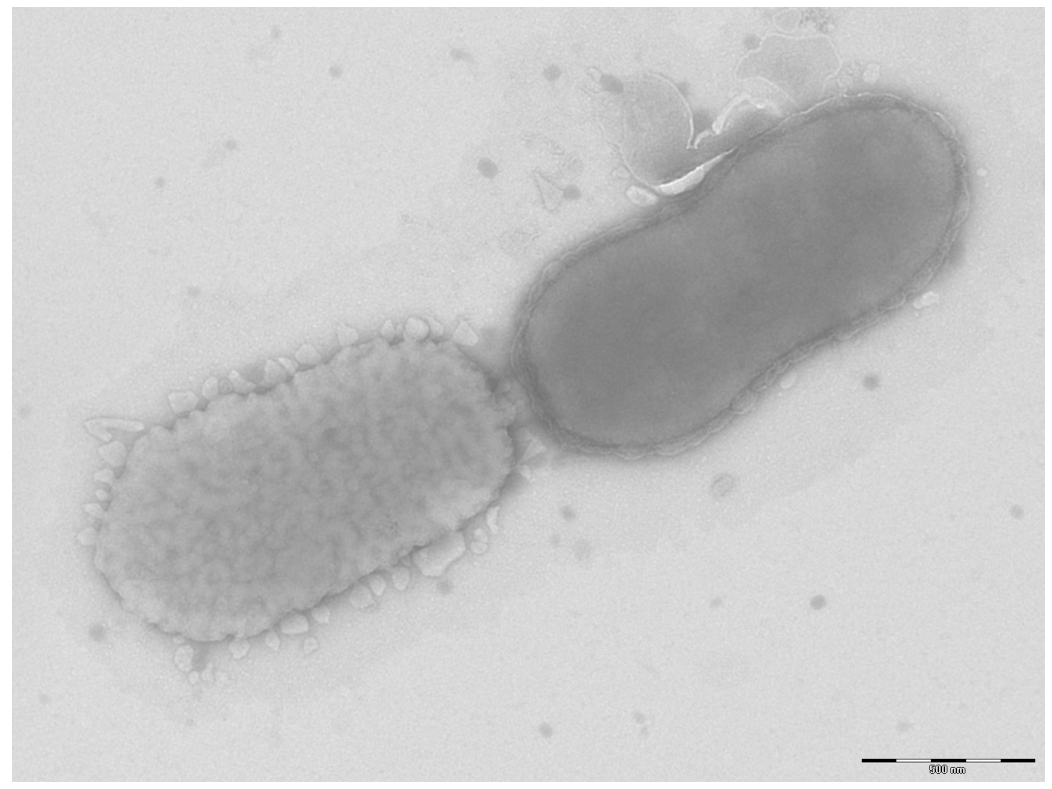

Figure 3. Transmission electron microscopy of $A$. timonensis strain JC136 ${ }^{\top}$, using a Morgani 268D (Philips) at an operating voltage of $60 \mathrm{kV}$. The scale bar represents $900 \mathrm{~nm}$.

Strain $136^{\mathrm{T}}$ exhibited catalase activity but no oxidase activity, and was resistant to $20 \%$ bile. Using API Rapid ID 32A, a positive reaction was obtained for $\alpha$-galactosidase, $\quad \beta$-galactosidase, $\beta$ glucuronidase, glutamic acid decarboxylase, leucyl glycine arylamidase and alanine arylamidase. Weak reactions were obtained for indole production and N-acetyl- $\beta$-glucosaminidase. No mannose and raffinose fermentation were observed. $A$. timonensis is susceptible to penicillin $\mathrm{G}$, amoxicillin + clavulanic acid, imipeneme, clindamycin, metronidazole and resistant to vancomycin. By comparison with $A$. senegalensis, strain $136^{\mathrm{T}}$ differed in mannose fermentation and proline arylamidase, arginine arylamidase and glycine arylamidase. By comparison with $A$. shahii, strain $136^{\mathrm{T}}$ differed in catalase activity and mannose and raffinose fermentation [7].

Matrix-assisted laser-desorption/ionization timeof-flight (MALDI-TOF) MS protein analysis was carried out as previously described [21]. Briefly, a pipette tip was used to pick one isolated bacterial colony from a culture agar plate, and to spread it as a thin film on a MTP 384 MALDI-TOF target plate (Bruker Daltonics, Leipzig, Germany). Four distinct deposits were done for strain JC136 from four isolated colonies. Each smear was overlaid with $2 \mu \mathrm{L}$ of matrix solution (saturated solution of alpha-cyano-4-hydroxycinnamic acid) in 50\% acetonitrile, $2.5 \%$ tri-fluoracetic-acid, and allowed to dry for five minutes. Measurements were performed with a Microflex spectrometer (Bruker). Spectra were recorded in the positive linear mode for the mass range of 2,000 to 20,000 Da (parameter settings: ion source 1 (IS1), $20 \mathrm{kV}$; IS2, $18.5 \mathrm{kV}$; lens, $7 \mathrm{kV}$ ). A spectrum was obtained after 675 shots at a variable laser power. The time of acquisition was between 30 seconds and 1 minute per spot. The four JC136 spectra were imported into the MALDI BioTyper software (version 2.0, Bruker) and analyzed by standard pattern matching (with default parameter settings) against the main spectra of 2,843 bacteria including the spectra from A. finegoldii, A. onderdonkii and A. shahii, used as reference data, in the BioTyper database. The method of identification included the $\mathrm{m} / \mathrm{z}$ from 3,000 to $15,000 \mathrm{Da}$. For every spectrum, 100 peaks at most were taken into account and compared with spectra in the database. A score enabled the identification, or not, from the tested species: a score $>2$ with a validated species enabled the identification at the species level, a score $>1.7$ but $<2$ enabled the identification at the genus level; and a score $<1.7$ did not enable any identification. For strain 136, the obtained score was 1.2, thus suggesting that our isolate was not a member of a known species. We incremented our database with the spectrum from strain JC136 (Figure 4). The spectrum was made available online in our free-access URMS database [22]. 


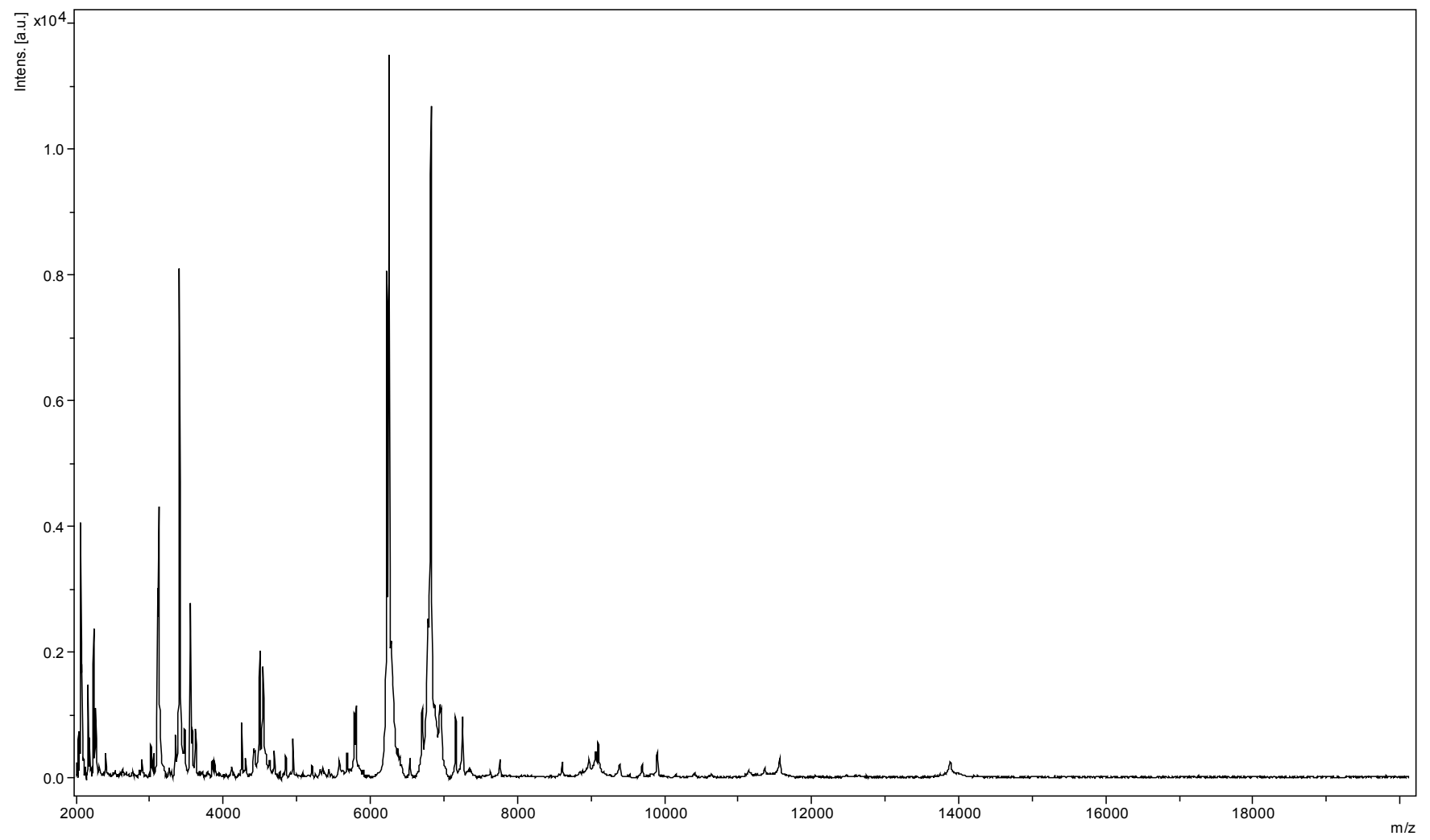

Figure 4. Reference mass spectrum from A. timonensis strain JC136 ${ }^{\top}$. Spectra from 4 individual colonies were compared and a reference spectrum was generated.

\section{Genome sequencing information}

\section{Genome project history}

The organism was selected for sequencing on the basis of its phylogenetic position and 16S rRNA similarity to other members of the genus Alistipes, and is part of a "culturomics" study of the human digestive flora aiming at isolating all bacterial species within human feces. It was the third genome of an Alistipes species and the first genome of Alistipes timonensis sp. nov. A summary of the project information is shown in Table 2. The EMBL accession number is CAEG00000000 and consists of 23 contigs. Table 2 shows the project information and its association with MIGS version 2.0 compliance [5].

\section{Growth conditions and DNA isolation}

A. timonensis sp. nov. strain JC136 ${ }^{\mathrm{T}}$, CSUR P148, DSM 25383, was grown anaerobically on 5\% sheep blood-enriched Columbia agar at $37^{\circ} \mathrm{C}$. Eight petri dishes were spread and resuspended in $4 \times 100 \mu \mathrm{l}$ of G2 buffer (EZ1 DNA Tissue kit, Qiagen, Hilden, Germany). A first mechanical lysis was performed by glass powder on the Fastprep-
24 device (MP Biomedicals, Santa Ana, CA, USA) using $2 \times 20$ seconds cycles. DNA was then treated with $2.5 \mu \mathrm{g} / \mu \mathrm{L}$ lysozyme for 30 minutes at $37^{\circ} \mathrm{C}$ and extracted using the BioRobot EZ 1 Advanced XL (Qiagen). The DNA concentration was measured at $40 \mathrm{ng} / \mu \mathrm{L}$ using the Genios fluorometer (Tecan, Lyon, France).

\section{Genome sequencing and assembly}

Both a shotgun and 3-kb paired-end sequencing were performed. The shotgun library was constructed with 500 ng of DNA with the GS Rapid library Prep kit (Roche). For the paired-end sequencing, $5 \mu \mathrm{g}$ of DNA was mechanically fragmented on a Hydroshear device (Digilab, Holliston, MA, USA) with an enrichment size at 3-4kb. The DNA fragmentation was visualized using the 2100 BioAnalyzer (Agilent, Massy, France) on a DNA labchip 7500 with an optimal size of 3.393 $\mathrm{kb}$. The library was constructed according to the 454 GS FLX Titanium paired-end protocol. Circularization and nebulization were performed and 
generated a pattern with an optimal size of 423 bp. After PCR amplification through 15 cycles followed by double size selection, the single stranded paired-end library was then quantified using the Genios fluorometer (Tecan) at $205 \mathrm{pg} / \mu \mathrm{L}$. The library concentration equivalence was calculated as $8,87 \mathrm{E}+08$ molecules $/ \mu \mathrm{L}$. The library was stored at $-20^{\circ} \mathrm{C}$ until further use.

The shotgun and paired-end libraries were clonally-amplified with $3 \mathrm{cpb}$ and $1 \mathrm{cpb}$, respectively, in $2 \times 8$ emPCR reactions with the GS Titanium SV emPCR Kit (Lib-L) v2 (Roche). The yields of the emPCR were $9.3 \%$ and $8.9 \%$, respectively. For each sequencing method, approximately 340,000 beads were loaded on the GS Titanium PicoTiterPlate PTP Kit $70 \times 75$ and sequenced with the GS FLX Titanium Sequencing Kit XLR70 (Roche). The run was performed overnight and then analyzed on the cluster through the gsRunBrowser and Newbler assembler (Roche). A total of 201,692 passed filter wells were obtained and generated $70.71 \mathrm{Mb}$ with a length average of $325 \mathrm{bp}$. The passed filter sequences were assembled using Newbler with $90 \%$ identity and $40 \mathrm{bp}$ as overlap. The final assembly identified 9 scaffolds and 23 contigs ( $>1,500 \mathrm{bp}$ ).

\section{Genome annotation}

Open Reading Frames (ORFs) were predicted using Prodigal [23] with default parameters but the predicted ORFs were excluded if they were spanning a sequencing GAP region. The predicted bacterial protein sequences were searched against the GenBank database and the Clusters of Orthologous Groups (COG) databases using BLASTP. The tRNAScanSE tool [24] was used to find tRNA genes, whereas ribosomal RNAs were found by using RNAmmer [25] and BLASTn against GenBank. ORFans were identified if their BLASTP $E$-value was lower than 1e-03 for alignment length greater than 80 amino acids. If alignment lengths were smaller than 80 amino acids, we used an $E$-value of 1e-05. Such parameter thresholds have already been used in previous works to define ORFans. To estimate the mean level of nucleotide sequence similarity at the genome level between Alistipes species, we compared the ORFs only using BLASTN and the following parameters: a query coverage of $>70 \%$ and a minimum nucleotide length of $100 \mathrm{bp}$.

\section{Genome properties}

The genome is $3,497,779$ bp long (one chromosome, no plasmid) with a $58.82 \%$ GC content (Table 3, Figure 5). Of the 2,742 predicted genes, 2,692 were protein-coding genes, and 50 were RNAs. A total of 1,885 genes $(70.02 \%)$ were assigned a putative function. Seventy-eight genes were identified as ORFans $(2.9 \%)$. The remaining genes were annotated as hypothetical proteins. The distribution of genes into COGs functional categories is presented in Table 4. The properties and the statistics of the genome are summarized in Tables 3 and 4.

Table 2. Project information

\begin{tabular}{lll}
\hline MIGS ID & Property & Term \\
\hline MIGS-31 & Finishing quality & High-quality draft \\
MIGS-28 & Libraries used & One paired end 3-kb library and one Shotgun library \\
MIGS-29 & Sequencing platforms & 454 GS FLX Titanium \\
MIGS-31.2 & Fold coverage & 20× \\
MIGS-30 & Assemblers & Newbler version 2.5.3 \\
MIGS-32 & Gene calling method & Prodigal \\
& EMBL ID & CAEG00000000 \\
& EMBL Date of Release & February 28, 2012 \\
& Project relevance & Study of the human gut microbiome \\
\hline
\end{tabular}


Table 3. Nucleotide content and gene count levels of the genome

\begin{tabular}{lrr}
\hline Attribute & Value & $\mathbf{\%}_{\text {of }}$ total $^{\mathbf{a}}$ \\
\hline Genome size (bp) & $3,497,779$ & \\
DNA coding region (bp) & $3,232,590$ & 92.42 \\
DNA G+C content (bp) & $2,057,393$ & 58.82 \\
Total genes & 2,742 & 100 \\
RNA genes & 50 & 2.0 \\
Protein-coding genes & 2,692 & 98.2 \\
Genes with function prediction & 1,885 & 70,0 \\
Genes assigned to COGs & 1,723 & 64.0 \\
Genes with peptide signals & 630 & 23.4 \\
Genes with transmembrane helices & 564 & 20.9 \\
\hline
\end{tabular}

a) The total is based on either the size of the genome in base pairs or the total number of protein coding genes in the annotated genome.

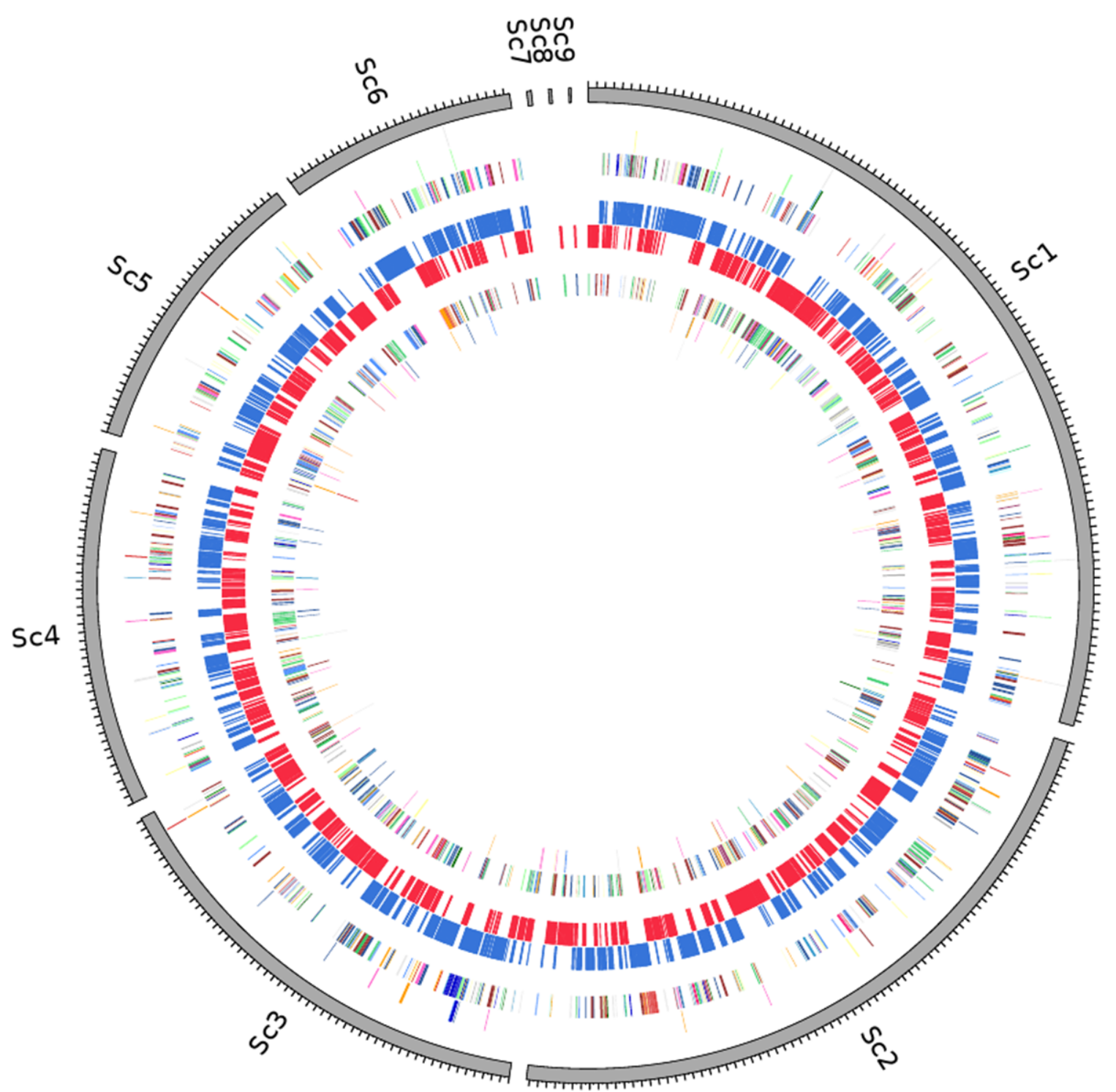

Figure 5. Graphical circular map of the chromosome. From outside to the center: Genes on forward strand (colored by COG categories), genes on reverse strand (colored by COG categories), RNA genes (tRNAs green, rRNAs red), GC content, and GC skew. 
Table 4. Number of genes associated with the 25 general COG functional categories

\begin{tabular}{crrl}
\hline Code & Value & \%age & Description \\
\hline J & 136 & 5.05 & Translation, ribosomal structure and biogenesis \\
A & 0 & 0 & RNA processing and modification \\
K & 124 & 4.60 & Transcription \\
L & 92 & 3.42 & Replication, recombination and repair \\
B & 0 & 0 & Chromatin structure and dynamics \\
D & 18 & 0.67 & Cell cycle control, mitosis and meiosis \\
Y & 0 & 0 & Nuclear structure \\
V & 31 & 1.15 & Defense mechanisms \\
T & 86 & 3.19 & Signal transduction mechanisms \\
M & 191 & 7.10 & Cell wall/membrane biogenesis \\
N & 6 & 0.22 & Cell motility \\
Z & 0 & 0 & Cytoskeleton \\
W & 0 & 0 & Extracellular structures \\
U & 32 & 1.19 & Intracellular trafficking and secretion \\
O & 62 & 2.30 & Posttranslational modification, protein turnover, chaperones \\
C & 112 & 4.16 & Energy production and conversion \\
G & 188 & 6.98 & Carbohydrate transport and metabolism \\
E & 141 & 5.24 & Amino acid transport and metabolism \\
F & 54 & 2.01 & Nucleotide transport and metabolism \\
H & 82 & 3.04 & Coenzyme transport and metabolism \\
I & 47 & 1.74 & Lipid transport and metabolism \\
P & 132 & 4.90 & Inorganic ion transport and metabolism \\
Q & 14 & 0.52 & Secondary metabolites biosynthesis, transport and catabolism \\
R & 234 & 8.69 & General function prediction only \\
S & 96 & 3.57 & Function unknown \\
- & 969 & 35.99 & Not in COGs \\
\hline & & & \\
\hline
\end{tabular}

The total is based on the total number of protein coding genes in the annotated genome.

\section{Comparison with other Alistipes genomes}

To date, the complete genomes from $A$. senegalensis strain JC50 ${ }^{\mathrm{T}}$ (GenBank accession number CAHI00000000), A. shahii strain WAL 8301 (GenBank accession number FP929032) and the unfinished genome from Alistipes sp. strain HGB5 (AENZ00000000) are available. A. timonensis has a smaller genome than $A$. senegalensis and $A$. shahii but a bigger genome than Alistipes sp. strain HGB5 $(3,497,779$ bp vs 4,017,609, 3,763,317 bp and $3,464,615$, respectively), a higher number of genes than $A$. shahii but smaller than $A$. senegalensis and Alistipes sp. strain HGB5 (2,742 vs 2,563, 3,163 and 2,955 genes, respectively), a higher ratio of genes assigned to COGs $(64.00 \%$ vs $58.56 \%, 58.9 \%$ and $62.53 \%$, respectively), and a higher $\mathrm{G}+\mathrm{C}$ content (58.82\% vs $57.33 \%, 58.4 \%$ and $57 \%$, respectively). In addition, $A$. timonensis shared mean nucleotide sequence similarities at the genome level of $92.18 \%$ (range 72.16 to $100 \%$ ), $88.72 \%$ (range 77.86 to $100 \%$ ) and $85.9 \%$ (range 77.4 to $100 \%$ ), with $A$. senegalensis strain $\mathrm{JC}^{\mathrm{T}}{ }^{\mathrm{T}}$, $A$. shahii strain WAL 8301 and Alistipes sp. strain HGB5, respectively. 


\section{Conclusion}

On the basis of phenotypic, phylogenetic and genomic analyses, we formally propose the creation of Alistipes timonensis sp. nov. that contains the strain JC136 ${ }^{\mathrm{T}}$. This bacterium has been cultivated from an healthy Senegalese individual, from whom was also cultivated $A$. senegalensis strain $\mathrm{JC}^{\mathrm{T}} \mathrm{T}^{\mathrm{T}}$, thus suggesting that the fecal flora from humans may contain several undescribed bacterial species that may be isolatable through diversification of culture conditions.

\section{Description of Alistipes timonensis sp. nov.}

Alistipes timonensis (tim.on.en'sis. L. gen. masc. n. timonensis, of Timone, the name of the hospital where strain JC136 ${ }^{\mathrm{T}}$ was isolated).

Colonies are 0.2 to $0.3 \mathrm{~mm}$ in diameter and produce brown pigment on blood-enriched Columbia agar and Brain Heart Infusion (BHI) agar. Cells are rodshaped with a mean diameter of $0.62 \mu \mathrm{m}$. Optimal growth is achieved anaerobically. No growth is observed in aerobic or microaerophilic conditions.

\section{References}

1. Rossello-Mora R. DNA-DNA reassociation methods applied to microbial taxonomy and their critical evaluation. In: Stackebrandt E (ed), Molecular Identification, Systematics, and population Structure of Prokaryotes, Springer, Berlin, 2006, p. 2350 .

2. Stackebrandt E, Ebers J. Taxonomic parameters revisited: tarnished gold standards. Microbiol Today 2006; 33:152-155.

3. Welker M, Moore ER. Applications of whole-cell matrix-assisted laser-desorption/ionization timeof-flight mass spectrometry in systematic microbiology. Syst Appl Microbiol 2011; 34:2-11. PubMed

http://dx.doi.org/10.1016/j.syapm.2010.11.013

4. Tindall BJ, Rossello-Mora R, Busse HJ, Ludwig W, Kämpfer P. Notes on the characterization of prokaryote strains for taxonomic purposes. Int I Syst Evol Microbiol 2010; 60:249-266. PubMed http://dx.doi.org/10.1099/ijs.0.016949-0

5. Rautio M, Eerola E, Vaisanen-Tunkelrott ML, Molitoris D, Lawson P, Collins MD, JousimiesSomer S. Reclassification of Bacteroides putredinis (Weinberg et al., 1937) in a new genus Alistipes gen. nov., as Alistipes putredinis comb. nov., and description of Alistipes finegoldii sp. nov., from human sources. Syst Appl Microbiol
Growth occurs between $30-37^{\circ} \mathrm{C}$, with optimal growth observed at $37^{\circ} \mathrm{C}$, in $\mathrm{BHI}$ medium $+5 \%$ $\mathrm{NaCl}$. Cells stain Gram negative and are non-motile. Catalase, $\alpha$-galactosidase, $\beta$-galactosidase, $\beta$ glucuronidase, glutamic acid decarboxylase, leucyl glycine arylamidase, $\mathrm{N}$-acetyl- $\beta$-glucosaminidase and alanine arylamidase activities are present. Indole production is also present. Oxidase activity is absent. Cells are susceptible to penicillin $\mathrm{G}$, amoxicillin + clavulanic acid, imipeneme and clindamycin and metronidazole. The $\mathrm{G}+\mathrm{C}$ content of the genome is $58.82 \%$. The $16 \mathrm{~S}$ rRNA and genome sequence are deposited in GenBank under accession numbers JF824799 and CAEG00000000, respectively.

A. timonensis is an obligate anaerobic Gramnegative bacterium. Grows on axenic medium at $37^{\circ} \mathrm{C}$ in an anaerobic atmosphere. Not motile.

The type strain $\mathrm{JC} 136^{\mathrm{T}}(=$ CSUR P148 = DSM 25383) was isolated from the fecal flora of a healthy patient in Senegal.

2003; 26:182-188. PubMed

http://dx.doi.org/10.1078/072320203322346029

6. Nagai F, Morotomi M, Watanabe Y, Sakon H, Tanaka R. Alistipes indinstinctus sp. nov. and Odoribacter laneus sp. nov., common members of the human intestinal microbiota isolated from faeces. Int J Syst Evol Microbiol 2010; 60:12961302. PubMed http://dx.doi.org/10.1099/ijs.0.014571-0

7. Song Y, Kononen E, Rautio M, Liu C, Bryk A, Eerola E, Finegold SM. Alistipes onderdonkii sp. nov. and Alistipes shahii sp. nov., of human origin. Int J Syst Evol Microbiol 2006; 56:19851990. PubMed http://dx.doi.org/10.1099/ijs.0.64318-0

8. Tyrrell KL, Warren YA, Citron DM, Goldstein EJ. Re-assessment of phenotypic identifications of Bacteroides putredinis to Alistipes species using molecular methods. Anaerobe 2011; 17:130-134. $\underline{\text { PubMed }}$ http://dx.doi.org/10.1016/j.anaerobe.2011.04.002

9. Fenner L, Roux V, Ananian P, Raoult D. Alistipes finegoldii in blood cultures from colon cancer patients. Emerg Infect Dis 2007; 13:1260-1262.

PubMed http://dx.doi.org/10.3201/eid1308.060662 
10. Saulnier DM, Riehle K, Mistretta TA, Diaz MA, Mandal D, Raza S, Weidler EM, Qin X, Coarfa C, Milosavljevic A, et al. Gastrointestinal microbiome signatures of pediatric patients with irritable bowel syndrome. Gastroenterology 2011; 141:1782-1791. PubMed http://dx.doi.org/10.1053/j.gastro.2011.06.072

11. Li F, Hullar MAJ, Schwarz Y, Lampe JW. Human gut bacterial communities are altered by addition of crucuferous vegetables to a controlled fruitand vegetable-free diet. J Nutr 2009; 139:16851691. PubMed http://dx.doi.org/10.3945/jn.109.108191

12. Torok VA, Hughes RJ, Mikkelsen LL, PerezMaldonado R, Balding K, MacAlpine R, Percy NJ, Ophel-Keller K. Identification and characterization of potential performance-related gut microbiotas in broiler chickens across various feeding trials. Appl Environ Microbiol 2011; 77:5868-5878. PubMed http://dx.doi.org/10.1128/AEM.00165-11

13. Woese CR, Kandler O, Wheelis ML. Towards a natural system of organisms: proposal for the domains Archae, Bacteria, and Eukarya. Proc Natl Acad Sci USA 1990; 87:4576-4579. PubMed http://dx.doi.org/10.1073/pnas.87.12.4576

14. Validation list $\mathrm{N}^{\circ} 143$. Int / Syst Evol Microbiol 2012; 62:1-4. http://dx.doi.org/10.1099/ijs.0.039487-0

15. Krieg NR, Ludwig W, Euzeby J, Whitman WB. Phylum XIV. Bacteroidetes phyl. nov. In: Krieg NR, Staley JT, Brown DR, Hedlund BP, Paster BJ, Ward NL, Ludwig W, Whitman WB (eds). Bergey's Manual of Systematic Bacteriology, Volume 4, Springer, New York 2011, p. 25.

16. Krieg NR. Class I. Bacteroidia class nov. In: Krieg NR, Staley JT, Brown DR, Hedlund BP, Paster BJ, Ward NL, Ludwig W, Whitman WB (eds). Bergey's Manual of Systematic Bacteriology, Volume 4, Springer, New York 2011, p. 25.

17. Krieg NR. Order I. Bacteroidales ord. nov. In: Krieg NR, Staley JT, Brown DR, Hedlund BP, Pas- ter BJ, Ward NL, Ludwig W, Whitman WB (eds). Bergey's Manual of Systematic Bacteriology, Volume 4, Springer, New York 2011, p. 25.

18. Krieg NR, Staley JT, Brown DR, Hedlund BP, Paster BJ, Ward NL, Ludwig W, Whitman WB. Family III. Rikenellaceae fam. nov. In: Krieg NR, Staley JT, Brown DR, Hedlund BP, Paster BJ, Ward NL, Ludwig W, Whitman WB (eds). Bergey's Manual of Systematic Bacteriology, Volume 4, Springer, New York 2011, p. 54.

19. List Editor. Validation List no. 94. Validation of publication of new names and new combinations previously effectively published outside the IJSEM. Int J Syst Evol Microbiol 2003; 53:17011702. PubMed http://dx.doi.org/10.1099/ijs.0.03001-0

20. Ashburner M, Ball CA, Blake JA, Botstein D, Butler H, Cherry JM, Davis AP, Dolinski K, Dwight SS, Eppig JT, et al. Gene ontology: tool for the unification of biology. The Gene Ontology Consortium. Nat Genet 2000; 25:25-29. PubMed http://dx.doi.org/10.1038/75556

21. Seng P, Drancourt M, Gouriet F, La Scola B, Fournier PE, Rolain JM, Raoult D. Ongoing revolution in bacteriology: routine identification of bacteria by matrix-assisted laser desorption ionization time-of-flight mass spectrometry. Clin Infect Dis 2009; 49:543-551. PubMed http://dx.doi.org/10.1086/600885

22. URMS database. http://ifr48.timone.univmrs.fr/portail2/index.php?option=com_content\&ta sk=view\&id=97 \&ltemid $=54$.

23. Prodigal. http://prodigal.ornl.gov

24. Lowe TM, Eddy SR. t-RNAscan-SE: a program for imroved detection of transfer RNA gene in genomic sequence. Nucleic Acids Res 1997; 25:955-964. PubMed

25. Lagesen K, Hallin P, Rodland EA, Staerfeldt HH, Rognes T, Ussery DW. RNAmmer: consistent and rapid annotation of ribosomal RNA genes. Nucleic Acids Res 2007; 35:3100-3108. PubMed http://dx.doi.org/10.1093/nar/gkm160 